\title{
Effects of high temperature annealing on the dislocation density and electrical properties of upgraded metallurgical grade multicrystalline silicon
}

\author{
XU HuaBi, HONG RuiJiang* \& SHEN Hui \\ Institute for Solar Energy Systems, State Key Laboratory of Optoelectronic Materials and Technologies, Sun Yat-sen University, Guangzhou \\ 510006, China
}

Received March 19, 2010; accepted June 12, 2010

\begin{abstract}
High temperature annealing was performed on upgraded metallurgical grade multicrystalline silicon (UMG multi-Si) wafers with a purity of $99.999 \%$. The samples were mechanically polished and chemically etched, and then the microstructures were observed by a scanning electron microscope (SEM). The minority carrier lifetime and resistivity of the samples were measured using microwave photoconductance decay and four-point probe techniques, respectively. The results show that the electrical properties of the samples decrease rather than increase as the annealing temperature increases, while the number of dislocations in bulk Si reduced or even disappeared after annealing for 6 hours at $1100-1400^{\circ} \mathrm{C}$. It is considered that the structural microdefects induced by the high concentration of metal impurities (including interstitial or substitutional impurities and nanoscale precipitates) determine the minority carrier recombination activity and thus the electrical properties of UMG multi-Si wafers rather than dislocations in bulk Si.
\end{abstract}

upgraded metallurgical grade multicrystalline silicon, high temperature annealing, dislocation density, minority carrier lifetime

Citation: $\quad \mathrm{Xu} \mathrm{H} \mathrm{B,} \mathrm{Hong} \mathrm{R} \mathrm{J,} \mathrm{Shen} \mathrm{H.} \mathrm{Effects} \mathrm{of} \mathrm{high} \mathrm{temperature} \mathrm{annealing} \mathrm{on} \mathrm{the} \mathrm{dislocation} \mathrm{density} \mathrm{and} \mathrm{electrical} \mathrm{properties} \mathrm{of} \mathrm{upgraded} \mathrm{metallurgical} \mathrm{grade}$ multicrystalline silicon. Chinese Sci Bull, 2011, 56: 695-699, doi: 10.1007/s11434-010-4090-7

Upgraded metallurgical grade silicon (UMG-Si) materials have received an increasing amount of attention recently because they have been recognized as a most promising substitute for high purity Si materials prepared by the traditional Siemens method. UMG-Si materials offer the advantage of a simple manufacturing process and low production cost for the photovoltaic industry [1-3]. At present, UMG-Si materials are commercially produced with a purity of $99.999 \%(5 \mathrm{~N})$, a production cost of less than $20 \mathrm{US} \$ / \mathrm{kg}$ and a conversion efficiency of more than $13.3 \%$. Improving the manufacturing process of UMG-Si materials will further increase the purity and reduce the production cost. Because of the high concentration of impurities $\left(>10^{18} \mathrm{~cm}^{-3}\right)$ such as $\mathrm{C}, \mathrm{O}, \mathrm{B}, \mathrm{P}, \mathrm{Fe}, \mathrm{Al}$ and $\mathrm{Ca}$ in UMG-Si solar cells, the aver-

*Corresponding author (email: hongruij@mail.sysu.edu.cn) age conversion efficiency is lower than that of high purity $(>7 N)$ Si solar cells by several percent. As a consequence, fabricating UMG-Si solar cells with a high average conversion efficiency has become a hot research topic in recent years.

The high concentration of impurities causes a large number of structural defects such as dislocations and impurity precipitates to exist in UMG-Si materials, especially multicrystalline Si (multi-Si) materials. Additionally, a high density of intergranular grain boundaries is present in UMG multi-Si materials. Such dislocations and grain boundaries are known to be decorated with impurity precipitates and act as sites of enhanced carrier recombination in bulk $\mathrm{Si}$, which significantly reduces the performance of UMG-Si solar cells [4-6]. A divergent issue is whether or not the dislocation density is the main factor that determines the 
carrier recombination activity among the structural defects and precipitates. Hartman et al. have ever reported that even relatively "clean" dislocations with weak individual recombination activity can have a large cumulative impact on minority carrier lifetime when present in the high concentrations typical of underperforming regions of multi-Si [7]. They believed that higher annealing temperatures $\left(>1170^{\circ} \mathrm{C}\right)$ would allow dislocation annihilation within minutes, suppressing minority carrier recombination activity. Ohshita et al. also reported that the grain boundaries did not play an important role in deteriorating the performance of solar cells; instead, the defects and impurities in grains determined the conversion efficiency. They particularly emphasized that many etch-pits (dislocations) that appeared during Secco etching acted as recombination centers and determined the minority carrier lifetime in low lifetime regions [8]. In this paper, high temperature annealing at the range of $1100-1400^{\circ} \mathrm{C}$ was performed on $5 \mathrm{~N}$ purity UMG-Si wafers to investigate if the dislocation density determined the minority carrier lifetime of the samples. The samples were mechanically polished and chemically etched so that the dislocations could be observed by scanning electron microscope (SEM). The lifetime and resistivity of the samples were determined.

\section{Experimental}

\subsection{Materials and instruments}

UMG multi-Si wafers (5N purity) were provided by Propower Renewable Energy (Shanghai) Co., Ltd. The mass percentages of the dominant impurities in the UMG multi-Si wafers are shown in Table 1 (provided by the wafer manufacturer), except for $\mathrm{C}$ and $\mathrm{O}\left(10^{17}-10^{18} \mathrm{~cm}^{-3}\right)$.

The main instruments used in the experiment include the following: a vacuum high-temperature tube furnace (GSL1600X, Hefei Kejing Materials Technology Co., China), a SEM (EVO-LS, Germany), a minority carrier lifetime tester (WT2000, SEMILAB, Hungary), and a four-point probe tester (RTS-9, Guangzhou 4Probes Electronic Technology Co., China) .

\subsection{Experimental procedure}

To investigate the effect of annealing at various temperatures on the dislocation density and electrical properties of UMG-Si samples, four neighboring Si wafers were selected so that the samples have approximately the same physical structure, chemical composition and electrical properties such as dislocation density, grain boundary, minority carrier lifetime, resistivity, concentration of impurities and their distribution of bulk $\mathrm{Si}$. One piece of the as-grown wafers was used as the control sample, the others were annealed for 6 hours at 1160,1260 and $1360^{\circ} \mathrm{C}$, respectively. The annealing conditions are shown in Figure 1. Nitrogen $\left(\mathrm{N}_{2}\right)$ was used to protect samples from oxidation during annealing. The as-grown and annealed samples were mechanically polished and then chemically etched in Sirtl etching solution (HF: $5 \mathrm{MCrO}_{3}$ ) for 30 seconds so that the dislocations in the wafers could be observed by SEM. The minority carrier lifetime of the samples was measured by the microwave photoconductance decay method and the resistivity was tested using the four-point probe technique.

\section{Results and discussion}

\subsection{Influence on dislocation density}

Three UMG multi-Si wafers were annealed for 6 hours under an $\mathrm{N}_{2}$ atmosphere at $1160,1260,1360^{\circ} \mathrm{C}$, respectively, while one wafer was left unannealed as a control. The as-grown and annealed samples were mechanically polished and chemically etched for $30 \mathrm{~s}$ using the Sirtl method. SEM was then employed to observe the dislocations in the samples. Figure 2 shows the statistical results for the dislocation density at a representative grain boundary after annealing at different temperatures. Figure 3 shows the SEM images of

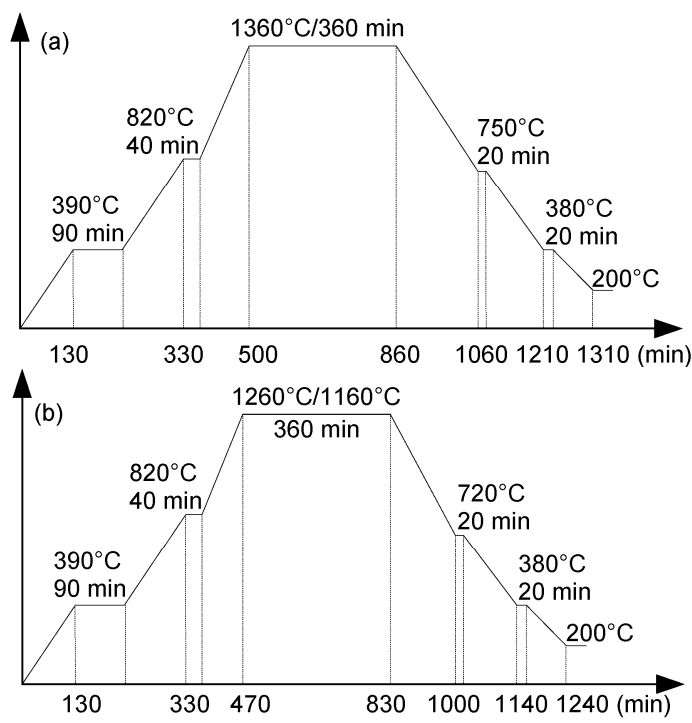

Figure 1 Annealing processes at temperatures of (a) $1360^{\circ} \mathrm{C}$; (b) 1160 and $1260^{\circ} \mathrm{C}$

Table 1 Dominant impurities present in UMG multi-Si wafers (\%)

\begin{tabular}{|c|c|c|c|c|c|c|c|c|c|}
\hline Impurity & B & $\mathrm{P}$ & $\mathrm{Fe}$ & $\mathrm{Al}$ & $\mathrm{Ca}$ & $\mathrm{Cu}$ & $\mathrm{Ni}$ & $\mathrm{Cr}$ & $\mathrm{Pb}$ \\
\hline Mass percent present $(\%)$ & 0.00032 & 0.0005 & 0.0002 & 0.0003 & 0.0002 & $<0.0001$ & $<0.0001$ & $<0.0001$ & $<0.0001$ \\
\hline
\end{tabular}


the etch-pits at the corresponding annealing temperatures. It can be seen that the etch-pit density decreases as the annealing temperature increases. When the annealing temperature was about $1360^{\circ} \mathrm{C}$, few etch-pits apart from the grain boundaries could be observed clearly. The results show that when the annealing temperature varies from 1100 to $1400^{\circ} \mathrm{C}$, the dislocation density in the bulk UMG multi-Si decreases as the annealing temperature increases, which is consistent with the experimental results of Hartman et al. [7].

It has been reported that metal precipitates at grain boundaries and dislocations can gradually dissolve and diffuse into the neighboring regions above $1000^{\circ} \mathrm{C}$ [9]. Generally, the rates of both dissolution and diffusion of metal impurities increase as the temperature is increased [10]. Upon dissolution and diffusion of metal precipitates, grain boundaries and dislocations in $\mathrm{Si}$ wafers can move within certain crystallographic glide planes at about $600^{\circ} \mathrm{C}$ [11]. As the temperature is increased above $1000^{\circ} \mathrm{C}$, dislocations can move unconstrained by glide planes and be removed from $\mathrm{Si}$ wafers via out-diffusion to surfaces or pairwise annihilation [12]. In view of this, the decrease in the dislocation density in UMG multi-Si bulk as the annealing temperature increases results from the dissolution and diffusion of metal precipitates at grain boundaries or intragranular dislocations and from the slippage of dislocations at high temperatures.

\subsection{Influence on electrical properties}

The minority carrier lifetimes of the samples were measured and results are shown in Figure 4. The resistivity of the samples was also measured; the results are shown in Figure 5 . When the annealing temperature is above $1100^{\circ} \mathrm{C}$, the lifetime and resistivity of the samples tend to decrease as the annealing temperature increases.

It should be mentioned that the concentration of impurities in the UMG multi-Si wafers did not changed during the

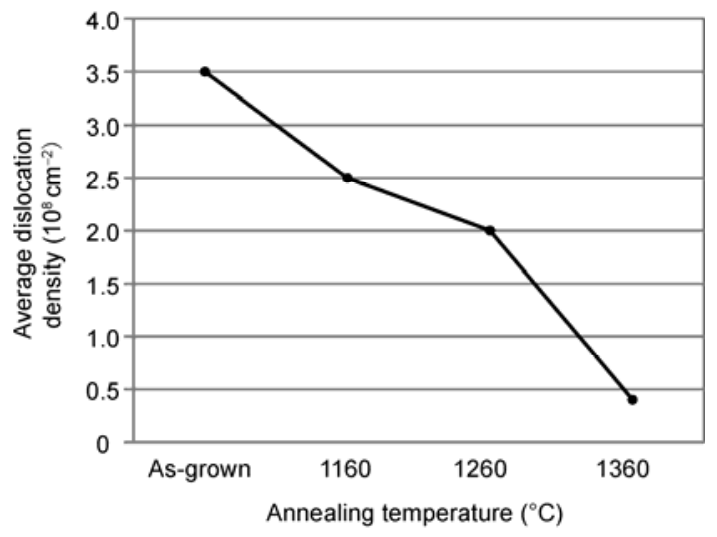

Figure 2 Average dislocation density of UMG multi-Si wafers at different annealing temperatures.
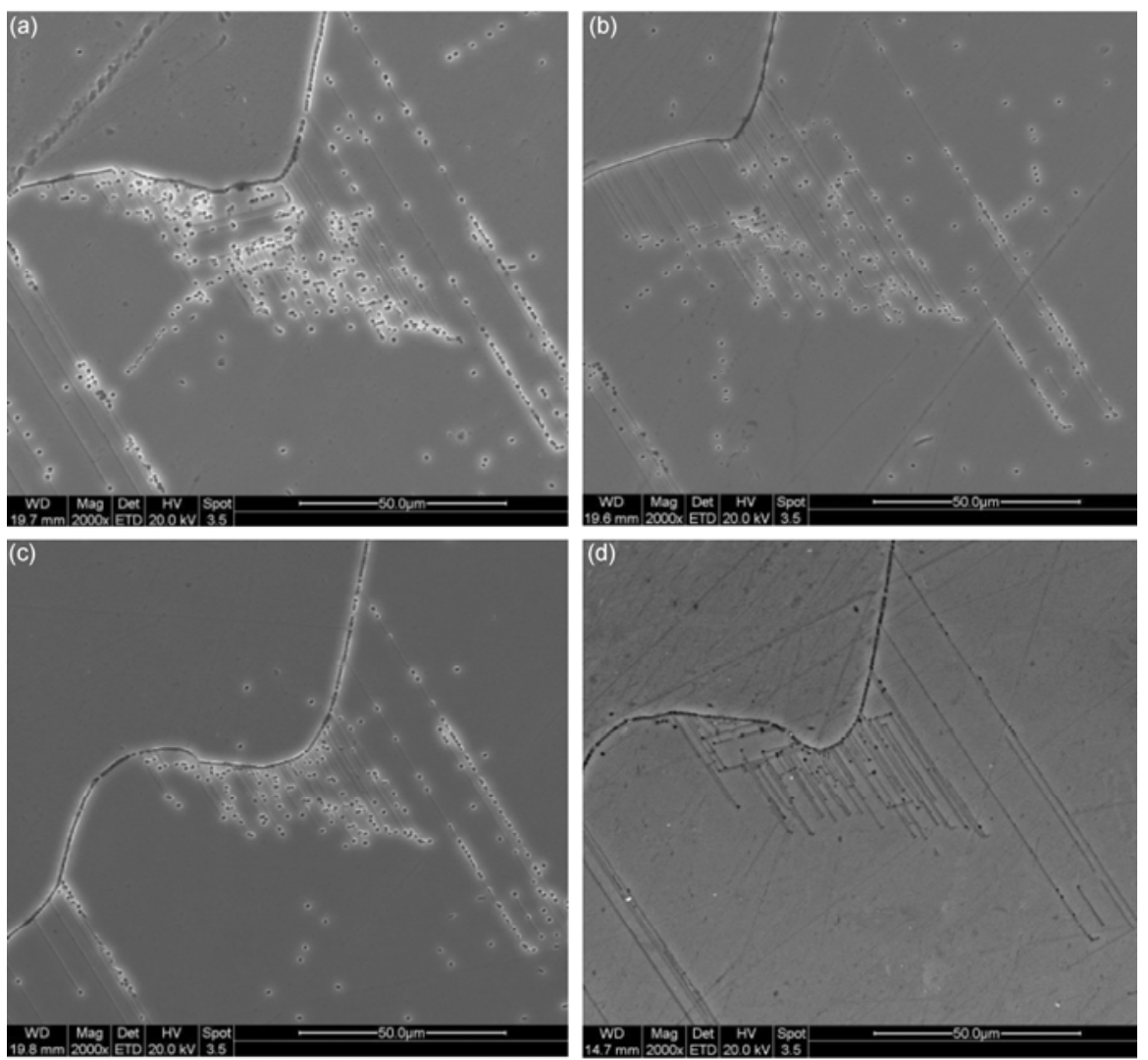

Figure 3 SEM images showing the dislocation density on UMG multi-Si wafers at different annealing temperatures $(\times 2000)$. (a) As-grown; (b) $1160^{\circ} \mathrm{C}$; (c) $1260^{\circ} \mathrm{C} ;(\mathrm{d}) 1360^{\circ} \mathrm{C}$. 


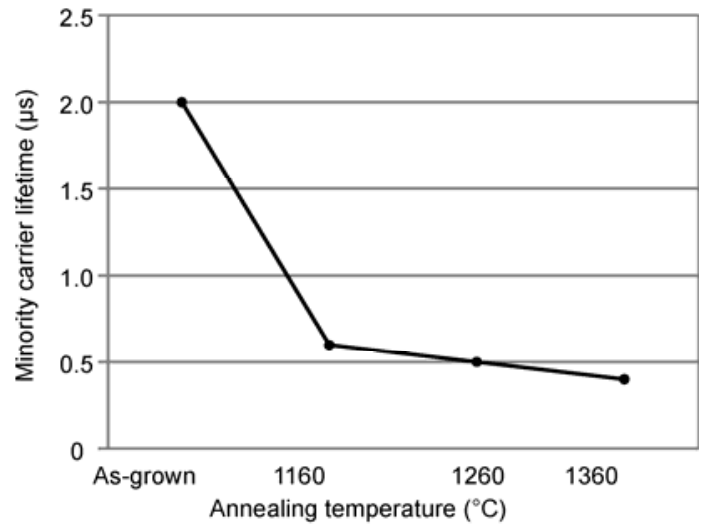

Figure 4 Minority carrier lifetime of UMG multi-Si wafers at different annealing temperatures.

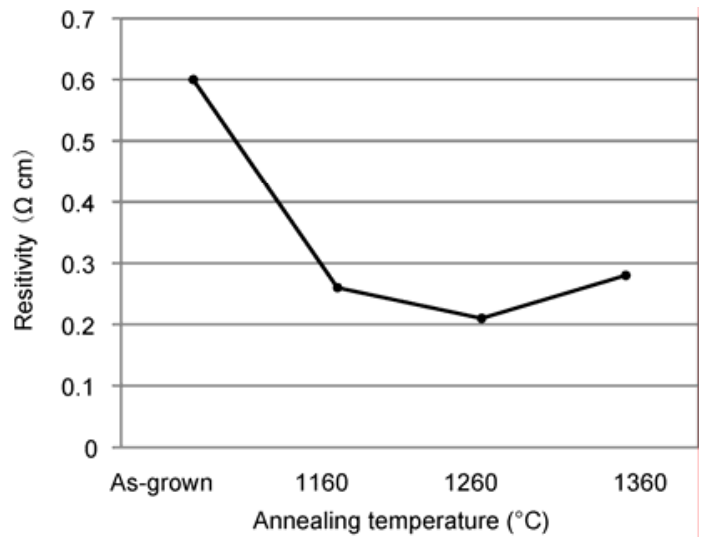

Figure 5 Resistivity of UMG multi-Si wafers at different annealing temperatures.

annealing process. Besides the decreases in the dislocation density, minority carrier lifetime and resistivity of the samples, the structure, chemical state and spatial distribution of the impurities in the samples may be changed by high temperature annealing. Istratov et al. reported that there is no significant difference in the concentration of metal impurities between regions with long and short lifetimes in the same $\mathrm{Si}$ wafer. When metal impurities conglomerate to form large precipitates at grain boundaries and dislocations in $\mathrm{Si}$ wafers, the precipitated impurities usually have low recombination activity. In contrast, the recombination activity of the metal impurities would be high enough to reduce the minority carrier diffusion length to less than $1 \mu \mathrm{m}$ if the impurities with high concentrations were in interstitial or substitutional sites [13-15]. It can be concluded that the properties of $\mathrm{Si}$ wafers and the resulting performance of solar cells not only depends on the total concentrations of impurities, but also on the structure, chemical state and spatial distribution of the impurities in the $\mathrm{Si}$ wafers. For example, $\mathrm{Fe}$ and $\mathrm{Cu}$ usually precipitate as silicides $\left(\mathrm{Fe}_{2} \mathrm{Si}\right.$ [9] and $\left.\mathrm{Cu}_{3} \mathrm{Si}[16]\right)$ at grain boundaries or dislocations. In addition, Buonassisi et al. have also reported that a large number of nanoscale precipitates formed during slow cooling from high temperature annealing that were widely and homogeneously distributed at intragranular microdefects. These nanoscale precipitates are usually formed of a mixture of silicides, oxides and carbides of different metal impurities and have much higher recombination activity than the microsized precipitates that conglomerated at grain boundaries and dislocations. As such, the nanoscale precipitates have a great influence on the electrical properties of Si materials [10]. In other words, in bulk Si there exist both microsized metal precipitates with low recombination activity that are inhomogeneously distributed at grain boundaries and dislocations, and nanoscale defects with high recombination activity that are widely distributed in the grains. To improve the properties of $\mathrm{Si}$ wafers, a suitable annealing temperature can be used to transfer some detrimental impurities into structures or chemical states with lower recombination activity. For instance, annealing in the range of $400-500^{\circ} \mathrm{C}$ can transfer most of the interstitial iron atoms into precipitates so that the electrical properties of the wafers can be improved [17].

For UMG multi-Si materials, the concentration of metal impurities is far higher than their solubility in bulk $\mathrm{Si}$ so most of the metal impurities precipitate or conglomerate inhomogeneously at grain boundaries and dislocations. Only about $1 \%-10 \%$ of the total impurities were found in the interstitial or substitutional sites and widely distributed in the grains. This small fraction of metal impurities usually has high recombination activity and thus has a decisive influence on the minority carrier diffusion length of Si materials. Only a slight variation in the structure or chemical state of metal impurities may significantly change the minority carrier recombination activity of Si materials, which is hardly dependent on the total concentration of impurities [9]. It is imagined that the high concentration of microscale precipitates or agglomerates at grain boundaries and dislocations can dissolve and diffuse into neighboring regions during high temperature annealing. The dissolved impurities are either widely distributed in the interstitial or substitutional sites or incorporated into the nanoscale precipitates at intragranular microdefects, which act as minority carrier recombination centers with high recombination activity and decrease the minority carrier lifetime of the wafers. The high concentration of metal impurities introduced into the grains during high temperature annealing contaminate the original "clean" grains and decrease the minority carrier lifetime and resistivity of the samples as the annealing temperature is increased.

The experimental results show that the minority carrier lifetime decreases rather than increases as the dislocation density in UMG multi-Si wafers is decreased. This finding is in conflict with the results of Hartman et al who reported that dislocation density had a decisive influence on the minority carrier lifetime of $\mathrm{Si}$ materials, especially multi-Si materials [7]. As well as the dislocation and grain boundary densities, the structure, chemical state and spatial distribu- 
tion of the metal impurities in the wafers have a significant or even decisive influence on the electrical properties of the UMG-Si materials, especially multi-Si materials with a high concentration of impurities. For high purity Si wafers with lower concentration of impurities $(>7 N)$, dislocation and grain boundary densities usually determine the minority carrier recombination activity [18].

\section{Conclusions}

Upon high temperature annealing of UMG multi-Si wafers with high concentrations of impurities at $1000-1400^{\circ} \mathrm{C}$, the dislocation density, minority carrier lifetime and resistivity of the wafers decreased as the annealing temperature increased. In other words, dislocation density is not the main limiting factor of the minority carrier lifetime of UMG multi-Si materials with a purity of $5 \mathrm{~N}$. Instead, the structure, chemical state and spatial distribution of the high concentration of metal impurities determine the minority carrier recombination activity of UMG-Si materials. Therefore, an optimal annealing temperature is required so that some detrimental impurities can be transferred into the structures or chemical states with lower recombination activity during the operation of UMG-Si solar cells.

This work was supported by the National Natural Science Foundation of China (50802118).

1 Gribov B G, Zinov'ev K V. Preparation of high-purity silicon for solar cells. Inorg Mater, 2003, 39: 653-662

2 Müller A, Ghosh M, Sonnenschein R, et al. Silicon for photovoltaic applications. Mater Sci Eng B, 2006, 134: 257-262

3 Bathey B R, Cretealla M C. Review: Solar-grade silicon. J Mater Sci 1982, 17: 3077-3096
4 Binetti S, Libal J, Acciarri M, et al. Study of defects and impurities in multicrystalline silicon grown from metallurgical silicon feedstock. Mater Sci Eng B, 2009, 159-160: 274-277

5 Taishi T, Hoshikawa T, Yamatani M, et al. Influence of crystalline defects in Czochralski-grown Si multicrystal on minority carrier lifetime. J Cryst Growth, 2007, 306: 452-457

6 Sopori B, Chen W, Tan T, et al. Overcoming the efficiency-limiting mechanisms in commercial Si solar cells. NCPV Photovolt Prog Rev, 1999: 341-347

7 Hartman K, Bertoni M, Serdy J, et al. Dislocation density reduction in multicrystalline silicon solar cell material by high temperature annealing. Appl Phys Lett, 2008, 93: 122108

8 Ohshita Y, Nishikawa Y, Tachibana M, et al. Effects of defects and impurities on minority carrier lifetime in cast-grown polycrystalline silicon. J Cryst Growth, 2005, 275: e491-e494

9 Buonassisi T, Istratov A A, Peters S, et al. Impact of metal silicide precipitate dissolution during rapid thermal processing of multicrystalline silicon solar cells. Appl Phys Lett, 2005, 87: 121918

10 Buonassisi T, Heuer M, Istratov A A, et al. Transition metal co-precipitation mechanisms in silicon. Acta Mater, 2007, 55: 6119-6126

11 Gumbsch P, Riedle J, Hartmaier A, et al. Controlling factors for the brittle-to-ductile transition in tungsten single crystals. Science, 1998, 282: 1293-1295

12 Takeuchi S, Argon A S. Review: Steady-state creep of single-phase crystalline matter at high temperature. J Mater Sci, 1976, 11: 1542-1566

13 Istratov A A, Buonassisi T, McDonald R J, et al, Metal content of multicrystalline silicon for solar cells and its impact on minority carrier diffusion length. J Appl Phys, 2003, 94: 6552-6559

14 Buonassisi T, Istratov A A, Pickett M D, et al. Chemical nature and distributions of metal impurities in multicrystalline silicon materials. Prog Photovolt: Res Appl, 2006, 14: 513-531

15 Buonassisi T, Istratov A A, Heuer M, et al. Synchrotron-based investigations of the nature and impact of iron contamination in multicrystalline silicon solar cells. J Appl Phys, 2005, 97: 074901

16 Buonassisi T, Marcus M A, Istratov A A, et al. Analysis of copper-rich precipitates in silicon Chemical state, gettering, and impact on multicrystalline silicon solar cell material. J Appl Phys, 2005, 97: 063503

17 Pickett M D, Buonassisi T. Iron point defect reduction in multicrystalline silicon solar cells. Appl Phys Lett, 2008, 92: 122103

18 Ehret E, Marty O. Correlation between electrical activity and extended defect in EMC multicrystalline materials. Mater Sci Eng B, 1998, 56: 24-30 